
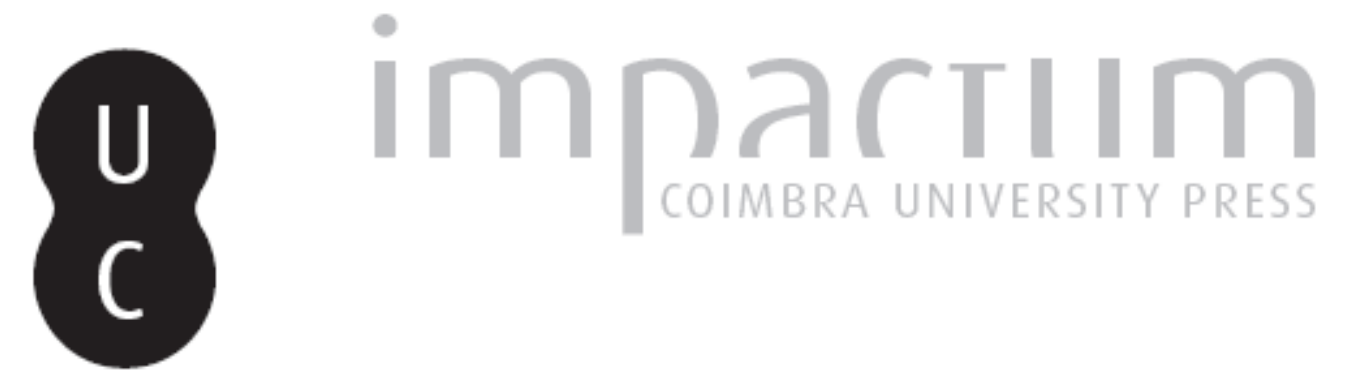

\title{
II Grillo di Plutarco e la tradizione della figura di Ulisse
}

\section{Autor(es): $\quad$ Casanova, Angelo}

Publicado por: International Plutarch Society

URL persistente:

URl:http://hdl.handle.net/10316.2/37623

DOI:

DOI:http://dx.doi.org/10.14195/0258-655X_4_2

Accessed : $\quad$ 26-Apr-2023 09:39:07

A navegação consulta e descarregamento dos títulos inseridos nas Bibliotecas Digitais UC Digitalis, UC Pombalina e UC Impactum, pressupõem a aceitação plena e sem reservas dos Termos e Condições de Uso destas Bibliotecas Digitais, disponíveis em https://digitalis.uc.pt/pt-pt/termos.

Conforme exposto nos referidos Termos e Condições de Uso, o descarregamento de títulos de acesso restrito requer uma licença válida de autorização devendo o utilizador aceder ao(s) documento(s) a partir de um endereço de IP da instituição detentora da supramencionada licença.

Ao utilizador é apenas permitido o descarregamento para uso pessoal, pelo que o emprego do(s) título(s) descarregado(s) para outro fim, designadamente comercial, carece de autorização do respetivo autor ou editor da obra.

Na medida em que todas as obras da UC Digitalis se encontram protegidas pelo Código do Direito de Autor e Direitos Conexos e demais legislação aplicável, toda a cópia, parcial ou total, deste documento, nos casos em que é legalmente admitida, deverá conter ou fazer-se acompanhar por este aviso.

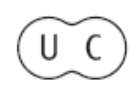


PLOUTARCHOS, n.S. Scholarly Journal of 18

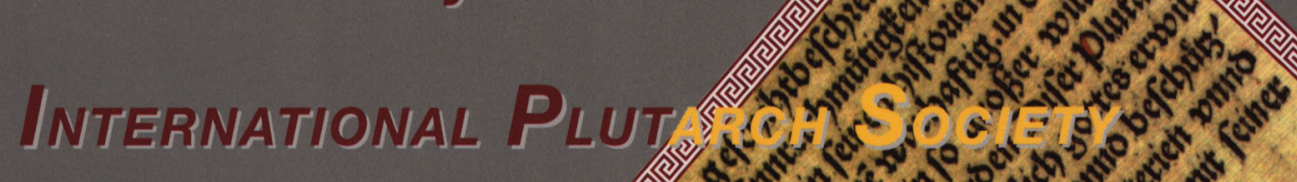

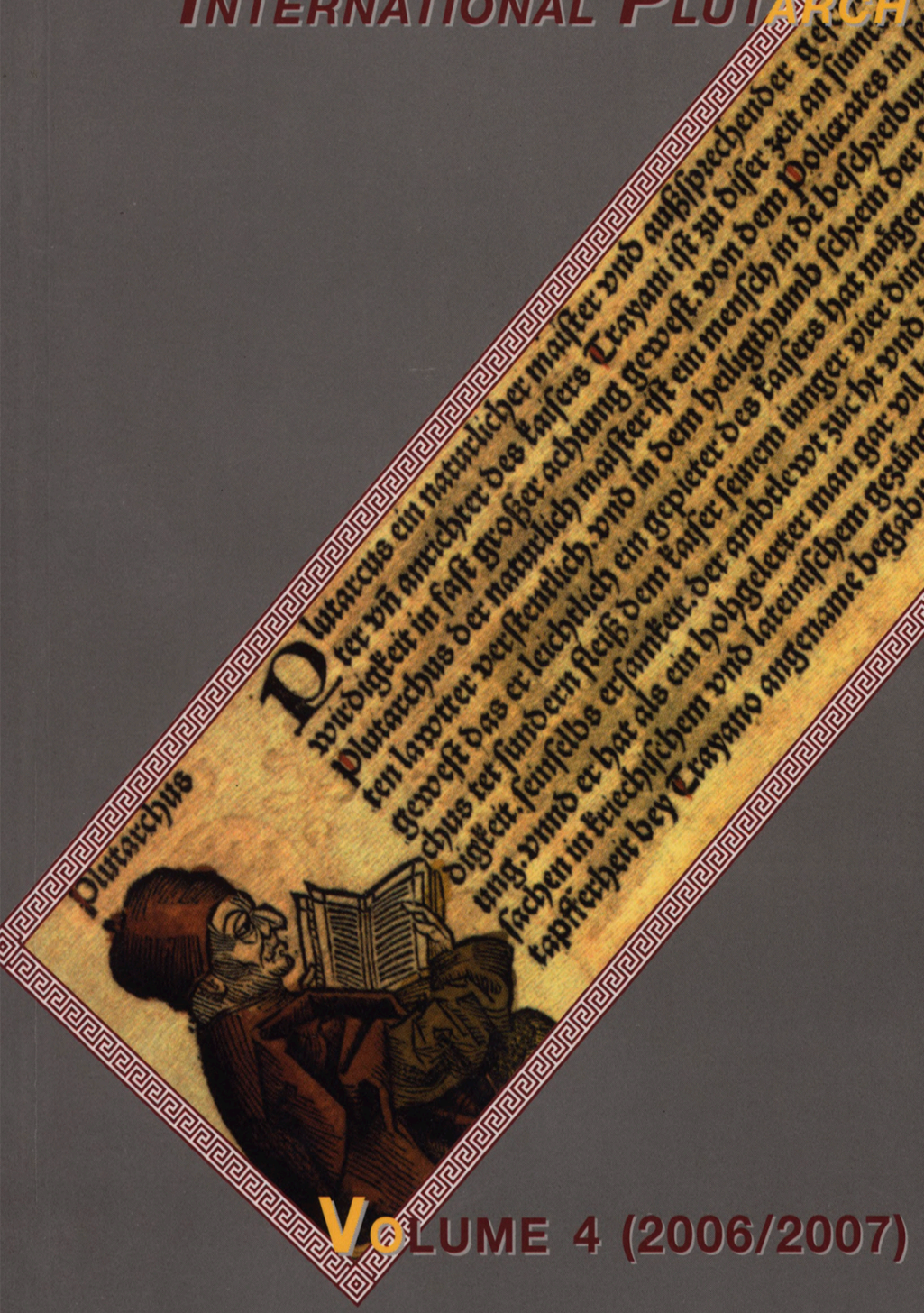

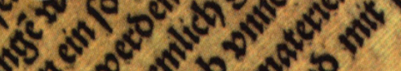

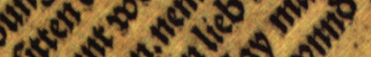

$120^{2} 0^{2} x^{2} x^{2}$

$4 \sin ^{\circ} \cos ^{\circ}$

singes

$0^{2}+0^{2} \mathrm{~s}^{\circ}$

$a^{5} x^{2}$

$s^{5}+\omega^{\circ}$

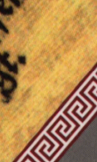

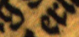

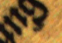




\title{
Il Grillo di Plutarco e la tradizione della figura di Ulisse* di \\ Angelo Casanova \\ Università di Firenze
}

\begin{abstract}
Plutarch's dialogue Bruta ammalia ratione uti develops the scene of Odissey XII, when Ulysses is about to leave Circe's island. But the hero's characterization by Plutarch, however sketchy, as well as Circe's and Gryllos' criticism of his behaviour are strongly reminiscent of Ulysses' figure as portrayed in the post-homeric tradition, particularly in the classical theatre, which constantly emphasized the hero's negative and pitiless sides, rather than his feats and his worth.
\end{abstract}

Nella mia relazione II Grillo di Plutarco e Omero, tenuta al "Séminaire Plutarque" di Lille (25-26 Novembre 2002) $)^{1}$ e nel volume di studi in onore di Philip StadteU ho presentato i risultati delle mie ricerche sulla ripresa di Omero nel dialogo di Plutarco Bruta animalia ratione uti, detto anche Grillo, dal nome del protagonista, un maiale che, parlando, tiene testa ad Ulisse e gli dimostra che è meglio essere animale che uomo.
In quelle pagine io ho argomentato che in questo curioso dialogo, dal contenuto paradossale, Plutarco riprende la scena dall'Odissea, e precisamente quella del canto XII (non quella del canto X, come di solito s'intende) ${ }^{3}$, quando Ulisse sta per partire, per ritornare in patria: e precisamente - quando Ulisse, dopo la cosiddetta visita all'oltretomba, ripassa nell'isola di Circe, solo per dare sepoltura al compagno Elpenore, e incontra la maga - con qualche timore - sulla spiaggia, presso la nave. Credo di aver dimo-

Relazione tenuta al VI Encuentro de la Red temàtica de Plutarco (Réseau thématique de Plutarque), Madrid, Universidad Complutense, 21-24 settembre 2005.

1

Ora inclusa nel volume di J. Boulogne (ed.), Les Grecs de l'antiquité et les animaux. Le cas remarquable de Plutarque, Lille, 2005, 97-109.

2 A. Casanova, "The Time Setting of the Dialogue Bruta animalia ratione uti", in: A. Pérez Jimenez \& F. Titchener (edd.), Historical and Biographical Values of Plutarch's Works. Studies devoted to Professor Philip A. Städter by the International Plutarch Society, Malaga- Logan (Utah), 2005, 121-131.

3 Cf. G. Indelli, 1995, p. 13 ss.; A. Zinato, 1995; M. Gondicas, 1998, p. 127 n. 1; G. Ditadi, 2000, p. 267; J. A. Fernandez Delgado, 2000, 171-181; D. Del Corno, intr. a D. MAGINI, 2001, p. $40 \mathrm{~s}$. 
strato che Plutarco, in questo dialogo, è più fedele ad Omero di quanto normalmente si dice tra gli studiosi.

Qui vorrei toccare brevemente un altro aspetto della figura di Ulisse nel $B A$, mettendo in luce $\mathrm{i}$ debiti che Pulisse di Plutarco ha verso la tragedia classica. La mia ricerca va dunque non alla fortuna di Plutarco, ma alla tradizione anteriore che Plutarco riprende.

Un elemento colpisce subito in maniera macroscopica iniziando la lettura del Grillo: Ulisse chiede a Circe se tra gli uomini da lei trasformati in lupi e leoni ci

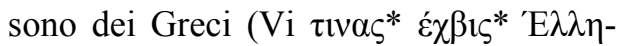
$\left.v \alpha \varsigma^{*}, 985 \mathrm{D}\right)$ e subito dopo chiarisce la motivazione: sarebbe per lui un bel motivo di

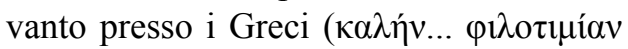
$\left.\pi \rho \circ \varsigma^{*} \tau o v \varsigma^{*}{ }^{r} \mathrm{E} \lambda \lambda \eta v \alpha \varsigma^{*}\right)$, se potesse portarli via, restituiti all'aspetto umano, sottraendoli ad un'esistenza così deplorevole e vergognosa. Proprio su questo tema si accenderà la discussione che fa da tema del dialogo. Quello che vorrei qui notare è la novità nella caratterizzazione di Ulisse: un condottiero decisamente ambizioso, interessato a liberare non solo i suoi compagni, ma addirittura tutti i Greci che possono essere lì, e non per filantropia, ma per $\varphi \imath \lambda o \tau \imath \mu i ́ \alpha$, cioè per desiderio di onore e gloria.

Ora, è ben noto a tutti che nelV Odissea Ulisse s'interessa alla salvezza sua e dei suoi compagni, che vuol riportare ad Itaca attraverso tutta la serie delle sue peripezie, salvandoli dai molteplici pericoli che incontrano nel viaggio. Mai - mi sembra- si accenna a desiderio di portare in salvo altri Greci. Tra l'altro, a questo punto delle sue peripezie (canto XII dell'Odissea, ripeto), ad Ulisse è rimasta una sola nave e con quella non potrebbe certo pensare di riportare in Grecia molta altra gente oltre i suoi.

Invece nel testo plutarcheo due elementi si delineano con chiarezza: la

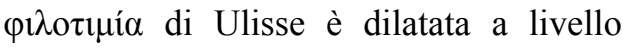
generale, panellenico, e per di più spicca l'uso della denominazione 'E $\lambda \lambda \eta v \alpha \varsigma$, che pone il discorso in termini propriamente anacronistici ${ }^{4}$. Non per caso Grillo, rispondendo in 988A, aderisce molto di più alla finzione cronologica del dialogo parlando di Cadmei, invece che di Tebani o Beoti.

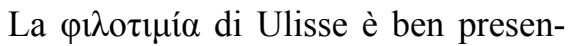
te in Omero, anzi è un tratto di carattere che Ulisse ha come ogni buon guerriero omerico, che notoriamente aspira ad essere $\alpha \rho \sigma \sigma \tau \varsigma^{*}$ e ad avere perciò non solo $\gamma \varepsilon \dot{\varepsilon} \rho \varsigma^{*}$ (cioè una assegnazione o parte di potere), ma anche fama, $\kappa \lambda \varepsilon \dot{c}$ $\varsigma^{*}$,

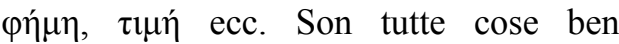
note $^{5}$. La cosa rilevante è che qui la $\varphi \imath \lambda o \tau \iota \mu i ́ \alpha$ di Ulisse è applicata vistosaeuropeo, trad. ital., Torino, 1963 , cap. 1; E. R. DodDS, I Greci e Firrazionale, trad. ital., Firenze, 2003 ( $1951^{1}$ ), capp. 1 e 2; W. JÀGER, Paideia, Firenze, 19532, voi. 1, cap. 1 -3; si veda da ultimo R. Di Donato, Aristeuein. Premesse antropologiche ad Omero, Pisa, 2006, passim. 
mente a tutti gli 'E $\lambda \lambda \eta v \alpha$, e dagli altri personaggi è subito connotata in senso negativo (da Circe, in $985 \mathrm{E}$ e in $986 \mathrm{~B}$, prima ancora che da Grillo).

Ora, io non credo che questo rovesciamento dipenda dal fatto che PUlisse del nostro dialogo possa essere inteso come un personaggio semiserio o paracomico, perché siamo nel mondo alla rovescia degli animali o perché siamo in una parodia... (come hanno argomentato Bergua Cavero, Femàndez Delgado e altri $)^{6}$. Per me la figura di Ulisse non è affatto comica, anche se la tesi del dialogo è paradossale. E che il gioco dei ruoli, nell'orchestrazione del dialogo, porta di necessità ad una contrapposizione tra Circe e, soprattutto, Grillo contro gli uomini tutti e contro Ulisse in particolare (date le sue ambiziose pretese) e, per la formulazione delle accuse contro di lui, i due personaggi attingono non solo ad Omero, ma a tutta la tradizione posteriore, e in particolare alla tragedia classica.

La figura di Ulisse 'criticabile', che compie imprese di vario tipo - anche di gusto discutibile o molto discutibile nell'interesse dei Greci tutti non è omerico, ma non è certo un'invenzione di Plutarco: è piuttosto una eredità della tragedia del V secolo.

In Omero Ulisse è mentitore e imbroglione, specie nell'Odissea (mentre $W Q \mid$ YIliade questo è un tema secondario, anche se non assente) $)^{7}$. È la ben nota caratterizzazione di Ulisse come "LUgner" e come "trickster". Ma è altrettanto notorio che tale caratterizzazione non attira censure etiche di rilievo da parte del poeta ; la sua astuzia è una virtù, un valore della mente, non qualcosa di riprovevole. L'Ulisse omerico è un furbo, un eroe vincitore che sa usare anche l'astuzia per vincere, non un tristo senza

Notoriamente Pulisse àzWIliade e quello delPOdissea non sono uguali, a cominciare da quella tiepidezza in combattimento che, attestata in II. 3.339 ss. e in 8.93 ss., scompare del tutto nell'altro poema. Viceversa, mentre nelVOdissea l'astuzia domina, essa $n z \mid Y$ Iliade è un tema secondario: cf. A. GARASSINO, 1930, p. 220.

8 E infatti, in $O d$. 9.19-20, l'eroe si autopresenta così: $\beta \mu{ }^{\prime} ' O \delta v \sigma \beta \hat{\zeta} \varsigma \Lambda \alpha \beta \rho \tau \imath \alpha ́ \delta \eta \varsigma, \quad \delta \varsigma$

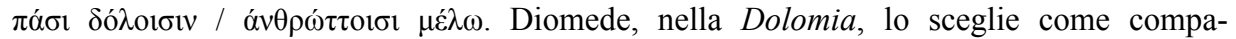

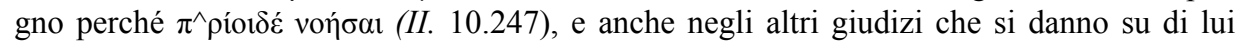

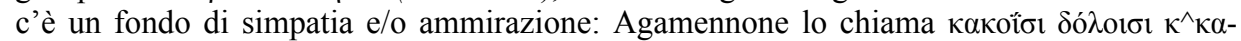

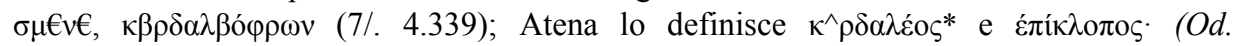

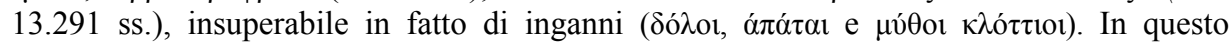
brano Atena parla del suo protetto come di un uomo in cui la logica della menzogna è connaturata e istintiva, poiché egli le ha parlato falsamente pur avendola riconosciuta sotto l'efebico travestimento. Tuttavia il passo non è da leggere in malam partem: si tratta di gioco e sfida d'abilità fra colleghi di бopía. Cf. ancora II. 3.202, dove Ulisse viene detto $\varepsilon i \delta \omega ́ \varsigma \pi \alpha v-$

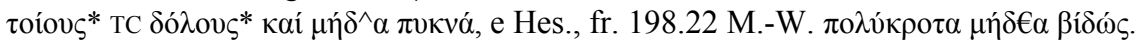


scrupoli; l'inganno gli serve per difendersi, o per risolvere problemi altrimenti insolubili, per riequilibrare rapporti di forza a lui sfavorevoli ${ }^{9}$. Egli usa l'inganno contro il gigantesco Polifemo, contro il numero soverchiante dei proci, contro

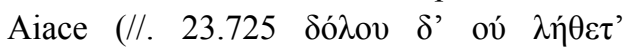

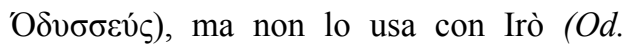
18.90-107), perché in questa lotta (una volta tanto) il più forte è lui. E prima, naturalmente, lo ha usato con la suprema invenzione del grande cavallo di legno per espugnare Troia (Od. 8.492 ss.).

Ma nel $B A^{10}$ non si parla mai deH'Ulisse 'mirabile' che vince usando anche l'astuzia, che inganna il Ciclope e i proci. Nelle parole di Circe e di Grillo emergono chiaramente rimproveri e accuse che noi conosciamo bene. L'Ulis- se attaccato da Grillo è quello che rovina Aiace, complotta contro Filottete, si sbarazza di Palamede.

Infatti Grillo dice di lui: "Noi non ti stimiamo affatto, perché è evidente che a torto avevi fama di intelligente e davi l'impressione di superare di gran lunga gli altri uomini nella capacità di pensare" (986C) e, più avanti (987C), scendendo sul terreno concreto del coraggio, lo mette sotto accusa: "Ne vai superbo e non nascondi il capo se ti chiamano temerario e distruttore

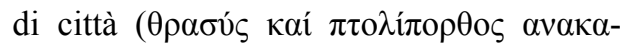
$\lambda$ ov́ $\mu \varepsilon v o \varsigma)$. Proprio tu, scelleratissimo, che dopo aver raggirato con insidie e artifici uomini esperti di un modo semplice e nobile di fare guerra, e ignari altresì di inganni e di menzogne, dai il nome di virtù alla tua malvagità" $(987 \mathrm{C})^{11}$.

Non è il solo che agisca così: alfinganno ricorre lo zoppo Efesto quando deve catturare il prestante Ares, "il più veloce degli dei" $\{O d .8 .330$ ss.); Nestore incoraggia Antiloco a usare l'astuzia nella gara dei carri, constatando che i cavalli altrui sono migliori (II. 23.311 ss.); sul tema cf. M. DETIENNE - J.-P. VERNANT, Le astuzie dell'intelligenza nell'antica Grecia, trad. it. Bari 1999.passim.

Altrove Ulisse è fatto oggetto di vari apprezzamenti da parte di Plutarco: vd. ad es. De adul. et am. 66F; Coniug. praec. 139A. Cf. W. B. STANFORD, 1954, pp. 158 n. 60, 270; BERGUA CAVERO, 1991, p. 17. Il differente giudizio su Ulisse espresso nel $B A$ rispetto ad altre opere non deve far pensare, come in altri casi, ad una vera e propria anomalia: bisogna tener presente che l'impostazione paradossale del dialogo porta a dare ampio spazio alla singolare opinione del parlante, dettata dal suo particolarissimo angolo di visuale.

11 Gli epiteti $\theta \rho \alpha \sigma u ́ \varsigma$ e $\pi \tau 0 \lambda i ́ \pi 0 \rho \theta o \varsigma$, in realtà, non sono i più tipici di Ulisse, che molto più

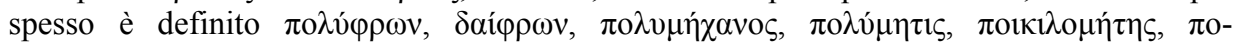

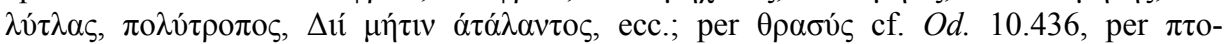
$\lambda i ́ \pi$ o $\theta$ o $\varsigma$ cf. II. 2.278, 10.363, ecc. Naturalmente Grillo ha una ragione per definirlo "distruttore di città", poiché all'epoca dei fatti Troia è già stata espugnata, e lo è stata

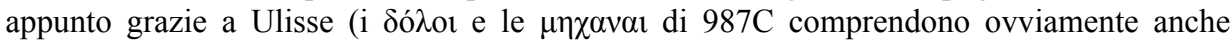

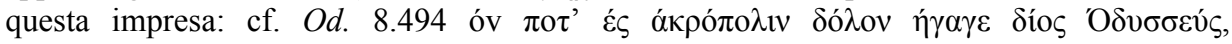
dove il $\delta$ ódo $\varsigma$ si riferisce appunto al cavallo di legno). Quanto a $\theta \rho \alpha \sigma u ́ \varsigma$, è notevole che l'unica occorrenza omerica di questo epiteto in relazione a Ulisse si trovi in Od. 10.436, che è appunto il canto di Circe. In questo passo, peraltro, $\theta \rho \alpha \sigma u ́ \varsigma$ è usato in senso peg- 
Qui è evidente che Grillo si riferisce non al modello epico omerico, ma ai tratti mitici ampliati e resi celebri dalla tragedia. Si tratta dunque di queU'Ulisse che recita piuttosto ruoli squallidi nel dramma attico $^{12}$, un Ulisse che non si batte contro i forti, ma infierisce sui deboli e i vinti, e in cui anche l'astuzia si è immeschinita, trivializzata, ed ha perso tutto ciò che aveva di grandioso e di magnanimo ${ }^{13}$. È quell'Ulisse che trovia- mo nel Filottete di Sofocle, miVEcuba di Euripide $^{14}$, ma viene ricordato anche nelle Troiane, nell'Ifigenia in Aulide di Euripide ${ }^{15}$ : e, aggiungiamo, svolgeva un ruolo di personaggio sostanzialmente 'odioso' anche in altre tragedie andate perdute, come nel Telefo, nel Filottete e nel Palamede di Euripide ${ }^{16}$ e, probabilmente, anche nelle omonime tragedie perdute di Eschilo, come anche nel $\mathrm{Pa}$ lamede e nel Telefo di Sofocle ${ }^{18}$.

giorativo ("disgraziato", "pazzo"), in riferimento ai fatti del Ciclope di 9.224 ss.; lo stes-

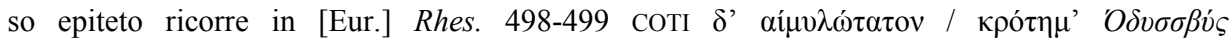

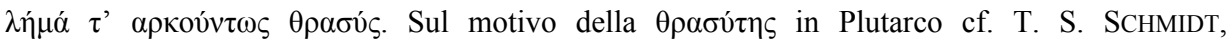
Plutarque et les barbares. La rhétorique d'une image, Louvain-Namur, 1999, pp. 69 ss.

E particolarmente in Euripide: cf. R. GoossENS, 1955, pp. 324 s. e 514 s.; nelYAiace sofocleo il personaggio è, come si sa, trattato meglio; per quanto riguarda la valutazione del giudizio espresso in Aesch., Ag. 841-844, gli studiosi discordano: cf. STANFORD, 1954, p. 103 e p. 261 n. 1, e R. AÉLION, Euripide héritier d'Eschyle, II, Paris, 1983, p. 333 n. 22.

Di questo fenomeno discute a lungo STANFORD, 1954, pp. 80-117, non senza rilevare (e con lui A. GARASSINO, 1930, p. 220) che le premesse di questa trasformazione dovevano trovarsi già in alcuni poemi pseudo-omerici e si dovrebbe perciò parlare di un Ulisse omerico e di un Ulisse tragico-ciclico. Come ha illustrato adeguatamente G. BASTA DONZELLI, 2001.

15 Poco importa che nelle Troiane e nella Ifigenia in Aulide egli non compare come personaggio agente sulla scena, ma di lui si parla ripetutamente, e proprio in termini di personaggio odioso.

Per il Telefo vd. la recente edizione di R. KAnNiCht, $\operatorname{Tr} G F$, voi. 5.2, p. 680 ss. (fr. 696 ss.); F. Jouan-H. van Looy, Euripide, tome Vili.3, Paris, 2002, p. 91 ss.; C. Preiser, Euripides: Telephos (Einleitung, Text, Commentar), Hildesheim, 2000. Per il Filottete, vd. $\operatorname{Tr} G F$, voi. 5.2, p. 827 ss. (fr. 787 ss.) K.; JouAN-VAN LoOY, ib., p. 269 ss.; G. AvezzÙ, Il ferimento e il rito. La storia di Filottete sulla scena attica, Bari, 1988, p. 124 ss. Per il Palamede, vd. $\operatorname{Tr} G F$ 5.2, p. 596 ss. (fr. 578 ss.); JoUAN-VAN LOOY, tome VIII.2, Paris, 2000, p. 487 ss.; R. FALCETTO, Il Palamede di Euripide, Alessandria, 2002.

17 Per i frammenti del Telefo di Eschilo vd. l'edizione di S. RADT, $\operatorname{Tr} G F$ 3, p. 343 ss. (fr. 238 ss.); per quelli del suo Filottete, p. 352 ss. (fr. 249 ss.); per quelli del suo Palamede, p. 295 ss. (fr. 181 s.).

18 Per i frammenti sofoclei, vd. l'edizione di S. RADT, $\operatorname{Tr} G F$ 4, p. 386 (Palamede), p. 434 (Telefo). Per i riferimenti al Filottete rimando alla recente edizione commentata della Fondazione Valla (Sofocle, Filottete, a cura di G. AvezzÙ e P. Pucci, trad. di G. CERRI, Milano, 2003). 
Nell'Aiace di Sofocle, come è noto, Ulisse fa una figura migliore. Ma, nel panorama generale della tragedia classica, non c'è dubbio che dal grande dolos eroico contro i mostri e le forze della natura si è passati alle trame e al basso intrigo di chi non esclude alcun mezzo per il raggiungimento del fine.

Aggiungiamo ancora che questo Ulisse non si limita a praticare il mendacio e fimbroglio, ma anche lo insegna ${ }^{19}$, ed è pertanto anche un corruttore. Maestro di falsità e raggiro, egli possiede platonicamente anche la scienza dell'opposto, e sa cosa si può e cosa non si può contraffare. Quando, in 987A, Grillo si aspetta da lui una bugia, Ulisse dice la verità, mettendo in luce la stessa diabolica bravura nel distinguere il кaıpó $\varsigma^{*}$ che egli dimostra in una celebre scena del Filottete sofocleo, dove così istruisce Neottolemo: "quando [Filottete] ti chiederà chi sei e da dove vieni, digli pure che sei figlio di Achille: su questo non devi

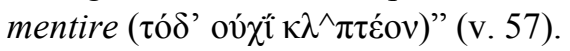

Lo sfondo della disputa non è più omerico: è quello della tragedia classica.

Questa distinzione fra Ulisse omerico e Ulisse tragico è ben nota ed acquisita, sia nella cultura comune che nei manuali di letteratura. Spesso però, sulla scorta di vecchi stereotipi tardoromantici, noi tendiamo a rappresentarci l'Ulisse del teatro attico come un caso da psicanalisi, un sadico, un artista del male. Ma vi sono forti argomenti che militano contro questa vulgata. Il principale è che $\mathrm{i}$ misfatti dell'Ulisse tragico, checché ne dicano a volte le sue vittime, non sono né gratuiti né ispirati da moventi personali.

Al contrario Ulisse agisce sempre, o quasi, nell'interesse della comunità ${ }^{22}$, interesse che egli deve talvolta perseguire anche contro il volere dei beneficati stessi, vincendo i loro scrupoli e sfidando la loro disapprovazione.

Non si può tuttavia argomentare che egli sia un semplice esecutore del volere dei capi, perché Ulisse è egli stesso un capo: anzi, morti Achille e Aiace, egli è (anche più di prima) tra le somme autorità del campo greco e, in particolare, il

Si veda il saggio di 'educazione sentimentale' che Ulisse somministra a Neottolemo in Soph. Ph. 96-109: quando ero giovane, egli dice, anch'io ero pronto a usare le mani piuttosto che la lingua, ma poi ho imparato che la lingua è più efficace; bisogna mentire, se il premio del mentire è il $\sigma \omega \theta \eta \dot{v} \alpha$.

"E bada di non irritarti e di non rispondermi contro l'evidenza, per amor di patria" ( $\pi \alpha \rho \alpha ́$

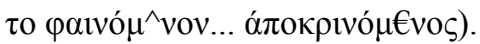

21 Scriveva già A. LESKY (Die tragische Dichtung der Hellenen, Gòttingen, 1972 , 246 = La poesia tragica dei Greci, Bologna, 1996, p. 366), che "sono in errore quegli studiosi che vedono nel personaggio di Odisseo un mezzo Mefistofele", perché "egli rappresenta piuttosto il servitore fedele dell'assemblea militare", di cui appare "strumento esecutivo": 
massimo trascinatore dell'impresa troiana nella sua fase finale, mente e braccio del potere, ministro e satellite dei capi ${ }^{23}$. Rispetto a questi (Agamennone e Menelao) egli non è né un suddito né un semplice sottoposto, poiché contribuisce attivamente a formare e orientare il loro volere, e talvolta persino lo crea. Egli non è neppure ciò che chiamiamo una 'eminenza grigia', poiché non delega ad altri l'esecuzione dei suoi piani, ma prende su di sé il lavoro sporco e si assume tutti i ruoli, da quello del consigliere a quello del boia, da quello del poliziotto a quello dell'aguzzino.

Poiché nella tragedia gli atti e i consigli di Ulisse sono sempre scellerati, è forte la tentazione di dare su di lui un giudizio liquidatorio, quasi che egli fosse più una maschera, un tipo, che un personaggio vero e proprio, e in quanto tale complesso e articolato. I malcapitati che ne diventano vittime lo definiscono spietato, persecutore, empio, vile, maramaldo ecc. ${ }^{24}$, e hanno tutte le ragioni per farlo. Ma il problema è che spesso anche gli studiosi moderni adottano questa indignata terminologia surrogandola all'autentica analisi critica.

In realtà questo bieco personaggio non può essere compreso se non si tiene presente il ruolo che la tragedia attica regolarmente gli assegna, il ruolo di eroe nazionale, difensore e tutore del popolo greco e della sua unità in qualunque situazione e ad ogni costo ${ }^{26}$; i delitti di Ulisse vengono compiuti in nome della patria e $\mathrm{d}$ dVethnos, e giustificati sulla base del principio che i nostri interessi, per il solo fatto di essere nostri, devono prevalere su ogni considerazione di pietà e di giustizia. 'E $\lambda \lambda \eta v \varepsilon \varsigma^{*}$ (985D-E e 986C), che è il vocabolo dell'unità, dell'identità (mentre più avanti, in 988a, Grillo aderisce di più alla finzione cronologica e parla di Cadmei invece che di Tebani o Beoti, come già segnalato a p. 20).

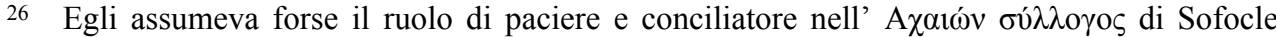
e nel Telefo di Euripide. Anche nell'Iliade Ulisse si presenta in questa veste, ad esempio allorché, nel libro II, si impegna a domare e a riunire l'esercito ammutinato (vv. 173 ss.), o allorché, nel libro IX, partecipa all'ambasceria che dovrebbe riportare concordia fra Achille e Agamennone (vv. 169 ss.). 
Nell'applicare questa logica perversa Ulisse è un isolato - ma isolato solo nella finzione letteraria: della sua figura si può dare una lettura politica decisamente importante. E gli si possono accostare personaggi ed episodi di guerra narrati da Tucidide. Gli Ateniesi compivano nella realtà molti degli atti che Ulisse compiva sulla scena, ed è illusorio credere che il pubblico seduto a teatro disapprovasse totalmente la condotta dell'eroe ${ }^{27}$; il Masqueray in fondo non aveva torto a dire che in Ulisse "la Grèce s'est incarnée presque entière".

L'anteporre un interesse di gruppo a una giustizia individuale non è altro che "Realpolitik", e perciò anche l'Ulisse tragico è un personaggio realpolitik . Egli però lo è alla sua maniera, poiché il suo concetto di 'interesse nazionale' non è solo pragmatico, ma anche, per così dire, concettuale: il buon nome e la fama della Grecia gli stanno a cuore tanto quanto i successi in guerra, il bottino, la prosperità. Anche questi obiettivi accessori, futili se vogliamo, meritano ai suoi occhi la rovina e il sacrificio altrui. L'eloquenza di
Ulisse, la sua abilità suasoria, entrano in gioco proprio per dimostrare che anche una crudeltà disumana è moralmente lecita qualora rechi a 'noi', a noi Greci, un benché piccolo vantaggio o piacere.

Anzi, ciò caratterizza pienamente la mentalità dell'Ulisse tragico, agli occhi del quale un piccolo vantaggio per 'noi' giustifica un grande crimine nei confronti di 'loro' (i nemici o avversari). Basti citare - uno per tutti - il caso deWEcuba euripidea, in cui Ulisse afferma la liceità etica di sgozzare una vergine barbara per onorare un eroe greco defunto: e consuona drammaticamente con quel punto del $B A$ in cui Grillo, il maiale parlante, accusa gli uomini di togliere agli animali quella grande cosa che è la vita per procurarsi un piccolo vantaggio quale è il piacere del palato ${ }^{30}$.

Ed è, ancora, una veste inconfondibilmente tragica quella in cui Ulisse ci appare nella frase pronunciata da Circe in 985E: "Ecco che uomo è costui! Nella sua stoltezza pretende che la propria ambizione si traduca in sventura non solo per sé e per i suoi compagni, ma anche

Sul problema del giudizio che il pubblico poteva dare sulla figura e sugli atti del personaggio si vedano le importanti osservazioni di G. BASTA DonZELLI, 2001; queste osservazioni riguardano YEcuba euripidea, che è il dramma in cui il problema si pone in maniera più acuta, ma valgono anche in generale.

P. MAsqueray, “Agamemnon, Ménélas, Ulyxe dans Euripide”, REA, 6 (1904) 173-204, p. 195.

29 Cf. GoOsSENS, 1955, p. 325.

30

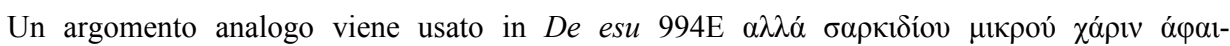

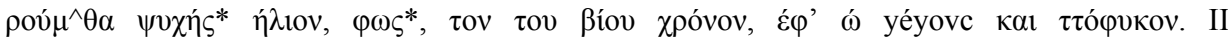
testo è evidentemente scorretto (ha cercato di sanarlo L. INGLESE, "Note critiche a Plutarco, De esu carnium", QUCC 44 (1993) 105-116, p. 107), ma il senso è chiaro. 
per chi gli è del tutto estraneo". Un uomo che non esita a sacrificare gli altri per la

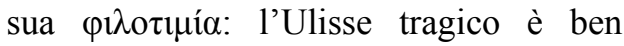
riconoscibile già da queste parole.

Un ultimo punto vorrei toccare, seppur brevemente, prima di concludere. Nel finale dell'opera, un finale notoriamente brusco, tanto brusco che diversi critici sospettano che il dialogo possa essere incompleto (ma questo è un problema in cui oggi non voglio entrare), di fronte all'obiezione finale di Ulisse che gli animali non hanno nozione della divinità, Grillo ribatte tagliente mettendo sotto accusa Ulisse in quanto figlio (o discendente) dell'ateo Sisifo. Questa fulminante battuta - a prescindere dalla completezza del dialogo - si spiega con riferimento ad una versione del mito sicuramente post-omerica, forse risalente ai Cypria 2, ma attestata soprattutto nella tragedia, sia in Eschilo (fr. 175 R., da $\mid V$ Armorum indicium) che in Sofocle (Ai. 190; Phil. 417, 625, 1311; fr. 567 R., dai Syndeipnoi) e in Euripide (/A 524 e 1362; Cycl. 104). Ed è una versione del mito ripresa e raccontata diffusamente da Plutarco, in $Q u$. Gr. 43, 301D. Secondo questa tradizione il vero padre di Ulisse non era Laerte, ma Sisifo, che avrebbe violentato Anticlea appena prima che sposasse Laerte $^{33}$. Sisifo, figlio di Eolo, nonno di Bellerofonte, era l'astuto per eccellenza, e si può capire il senso di questo ardito incrocio mitico che spiega l'astuzia di Ulisse come eredità patema. Ma Sisifo era anche il grande ateo, condannato nell'Ade ad un supplizio esemplare (il supplizio di Sisifo è già noto ad Omero: $O d$. 11.593 ss.). E il tema dell'ateismo di Sisifo fu sviluppato in particolare in un'altra tragedia, nel Sisifo di Crizia: vd. $\operatorname{Tr} G F$ I, 43 fr. 19, un ampio frammento citato da Sesto Empirico, dove si sosteneva che gli dèi sarebbero l'invenzione di qualche uomo astuto per incutere timore ai malvagi.

Dunque il finale del Grillo contiene una grossa cattiveria contro Ulisse, una battuta velenosa: e bisogna intendere non "discendente di Sisifo", ma "nato da Sisifo", o come dice IA 524 "seme di

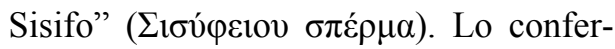
ma il brano plutarcheo delle $Q u$. $G r$. $301 \mathrm{D}$, per quanto si presenti come un semplice appunto, o la rapida annotazione di una curiosità erudita.

OPERE PIÙ VOLTE CITATE

BASTA DONZELLI, G.,

- 'Odisseo neWEcuba di Euripide", Lexis,

19 (2001) 185-197.
Cf. e.g. K. ZIEGLER, 1965, p. 131; G. InDELli, 1995, p. 138; e D. DEL CoRNO nella introduzione a D. MAGINI, 2001, p. 42.

Cf. F. Jounn, Euripide et les légendes des Chants Cypriens, Paris 1966, p. 279; e Euripide VILI, Iphigénie à Aulis, Paris, 1983, p. 134.

In $Q u$. Gr. 43, 301D Plutarco cita Istro per un particolare, ma aggiunge che questa ver-

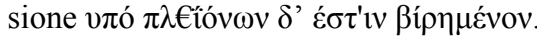


Bergua CAVERo, J.,

- "Cinismo, ironia y retòrica en el Bruta ratione uti de Plutarco", in J. GARCÌA LOPEZ \& E. CALDERÓN DORDA (edd.), Estudios sobre Plutarco: paisajey naturaleza, Actas del II Simposio Espanol sobre Plutarco, Murcia 1990, Madrid, 1991, 13-19.

Del Corno, D.: vd. Magini, D.

DITADI, G,

- Plutarco. L'intelligenza degli animali e la giustizia loro dovuta, Este, 2000.

FERnÀndez Delgado, J. A.,

- "Le Gryllus, une éthopée parodique", in L. VAN DER Sтоскт (ed.), Rhetorical Theory and Praxis in Plutarch, A età of thè $I V^{* \prime \prime}$ International Congress of thè International Plutarch Society, Leuven, july 3-6, 1996, Louvain-Namur, 2000, 171-181.

GARASSINO, A.,

- "Ulisse nel teatro greco", $A \& R, 2$ (1930) 219-251.

GONDICAS, M.,

- Plutarque. L'intelligence des animaux; suivi de Gryllos, trad. du grec et présen- té par M. G, Paris, 1998.

GoOsSENS, R.,

- Euripide et Athènes, Bruxelles, 1955.

INDELLI, G,

Plutarco. Le bestie sono esseri razionali, intr., testo cr., trad. e comm. a c. di G. L, Napoli, 1995.

Magini, D.,

- Plutarco. Del mangiar carne. Trattati sugli animali, trad. e note di D.M., con intr. di D. Del CoRno, Milano, 2001.

STANFORD, W. B.,

- The Ulysses Theme. A Study in thè Adaptability of a Traditional Hero, Oxford, $1954\left(1963^{2}\right)$.

ZlEGLER, K.,

Plutarco, trad. it., Brescia 1965 (= "Plutarchos von Chaironeia", $R E \mathrm{XX} 1.1$, 1951, coll. 636-962).

zinato, A.,

Plutarco. Le virtù degli animali, a c. di A. Z., con introduzione di O. Longo, Venezia, 1995. 